\title{
Conflict monitoring engages the mediofrontal cortex during nonword processing
}

\author{
Markus J. Hofmann ${ }^{\mathrm{a}}$, Sascha Tamm ${ }^{\mathrm{a}}$, Mario M. Braun ${ }^{\mathrm{a}}$, Michael Dambacher ${ }^{\mathrm{b}}$, Anja Hahne \\ and Arthur M. Jacobs ${ }^{\mathrm{a}}$
}

\author{
aDDepartment of Psychology, Free University Berlin, ${ }^{\mathrm{b}}$ Department of Psychology, University of Potsdam, Potsdam and ${ }^{\mathrm{C}}$ Max-Planck Institute for \\ Human Cognitive and Brain Sciences, Leipzig, Germany \\ Correspondence to Markus J. Hofmann, Department of Psychology, Free University Berlin, Habelschwerdter Allee 45, 14195 Berlin, Germany \\ Tel: + 4930 83855633; e-mail: mhof@zedat.fu-berlin.de
}

Received 19 October 2007; accepted 23 October 2007

\begin{abstract}
The current study investigated the role played by conflict monitoring in a lexical-decision task involving competing word representations, using event-related potentials. We extended the multiple read-out model (Grainger and Jacobs, 1996), a connectionist model of word recognition, to quantify conflict by means of Hopfield Energy, which is defined as the sum of the products of all orthographic word node pair activations within the artificial mental lexicon of this model. With increasing conflict levels in nonwords,
\end{abstract}

a late negativity increased in amplitude $(400-600 \mathrm{~ms})$ accompanied by activation of the anterior cingulate cortex and the medial frontal gyrus. The simulated conflict predicted the amplitudes associated with this mediofrontal conflict-monitoring network on an item level, and is consistent with the conflict-monitoring theory.

Keywords: conflict-monitoring theory, event-related potentials, multiple read-out model, standardized low-resolution brain electromagnetic tomography, word recognition

\section{Introduction}

Monitoring of conflicting bits of information is an essential human ability, to respond flexibly to the environment. Many neuroimaging studies associate conflict monitoring with mediofrontal brain areas such as the anterior cingulate cortex [1] and the medial frontal gyrus [2]. The aim of this study is to demonstrate the role of conflict monitoring during the processing of letter stimuli. We show that a connectionist model of lexical processing can account for behavioral and event-related potential (ERP) responses that are related to mediofrontal networks.

The multiple read-out model (MROM) [3,4] was used to make predictions about human performance in the lexicaldecision and related letter-string processing tasks. It basically contains a feature, a letter, and a word level, with the lower levels providing inputs to the higher levels (see Fig. 1 and Ref. [4] for further details). For example, when the letter $\mathrm{F}$ is encoded at the feature level, the letter node $\mathrm{F}$ becomes activated to a larger degree than the letter node B, whereas letter nodes that share no feature with $\mathrm{F}$ remain inactivated. The same is true for the relationship between the letter and the word levels. The nonword FLUR thus activates the word node BLUR to a larger degree than the word node BLUE, as it shares more letters with this node.

Botvinick et al. [1,5] reviewed converging evidence that longer response times, higher error rates, and larger anterior cingulate cortex activations were observed in response to partially activated and conflicting response alternatives in the Eriksen flanker and Stroop tasks. More importantly,
Botvinick et al. [1] used a very similar framework as the MROM (see Fig. 1) to make predictions about the extent of conflict during a stem completion task in which participants had to complete a word stem (e.g. BLU_) to form a whole word (e.g. BLUR or BLUE). The simulated amount of conflict between the word nodes accounted for the finding that the so-called strength ratio predicts anterior cingulate cortex activity [6]. This ratio is calculated by dividing the frequency of the most frequent completion by the frequency of the second most frequent completion. The larger the competition that exists between these completions, the larger will be the conflict itself, and thus the anterior cingulate cortex activity. As lexical frequency determines the resting levels of the word nodes in the MROM, it should be capable of accounting for ratio effects, when a measure of conflict between the word nodes is introduced. This is very similar to Grainger and Jacobs' [3] lexical inhibition hypothesis predicting greater response times and error rates for nonwords during lexical decision, with an increasing number and relative activations of the word nodes partially activated by a nonword.

Botvinick et al. [1] predicted larger anterior cingulate cortex activations in response to the larger conflict between activated word nodes. The aim of this study was to test the hypothesis, that the keener the competition between these word nodes, the larger will be the anterior cingulate cortex [1,7] and medial frontal gyrus activity [2]. In ERPs, larger anterior cingulate cortex and medial frontal gyrus activities are often associated with the N2 component being most 


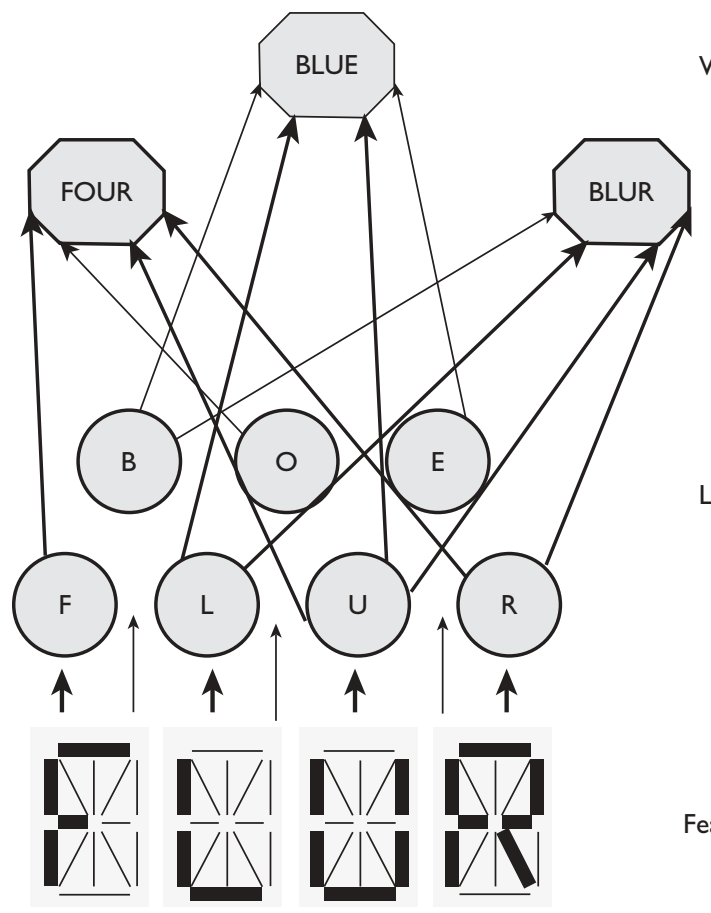

Fig. I Schematic representation of the basic architecture of the multiple read-out model (MROM). If more features of a letter are activated, there is greater activation of the respective letter node at the letter level. If more letters of a word node are activated, more activation is fed forward to the respective word nodes. The relative activation of the nodes and the strength of the feed-forward activations are indicated by line thickness.

prominent at frontal sites [7]. Therefore, we conducted an ERP study using a lexical-decision task, and applied standardized low-resolution brain electromagnetic tomography (sLORETA) for source localization [8]. Botvinick et al. [1] quantified the extent of conflict by means of the so-called Hopfield Energy (Ehopf), which is the sum of the products of all possible pairs of word node activations. For example (see Fig. 1), FLUR activates the word node pairs BLUE and BLUR, BLUE and FOUR, and FOUR and BLUR. We implemented Ehopf into the MROM without changing parameters (version of Ref. [9]). It includes most of the three-letter to five-letter German monosyllabic words, in all 1025 words. Ehopf was computed from cycles 2-7 [9] and $\log$ transformed for normal distribution purposes. Two items were excluded from item analyses because they revealed an Ehopf of zero, resulting in missing values after $\log$ transformation.

\section{Methods}

Fourteen right-handed German native speakers (mean age 23 years, range 19-30, seven women) were recruited from the Max-Planck Institute for Human Cognitive and Brain Sciences and were paid for their participation. They had normal or corrected-to-normal vision.

Stimuli were four-lettered, and consisted of 300 German words and 300 nonwords. Nonwords included a large range of generable nonwords from nonpronounceable consonant strings (e.g. GKNZ) that activate only one word node (GANZ) to pronounceable nonwords (e.g. LUND), which activate many word nodes (e.g. RUND, LAND,
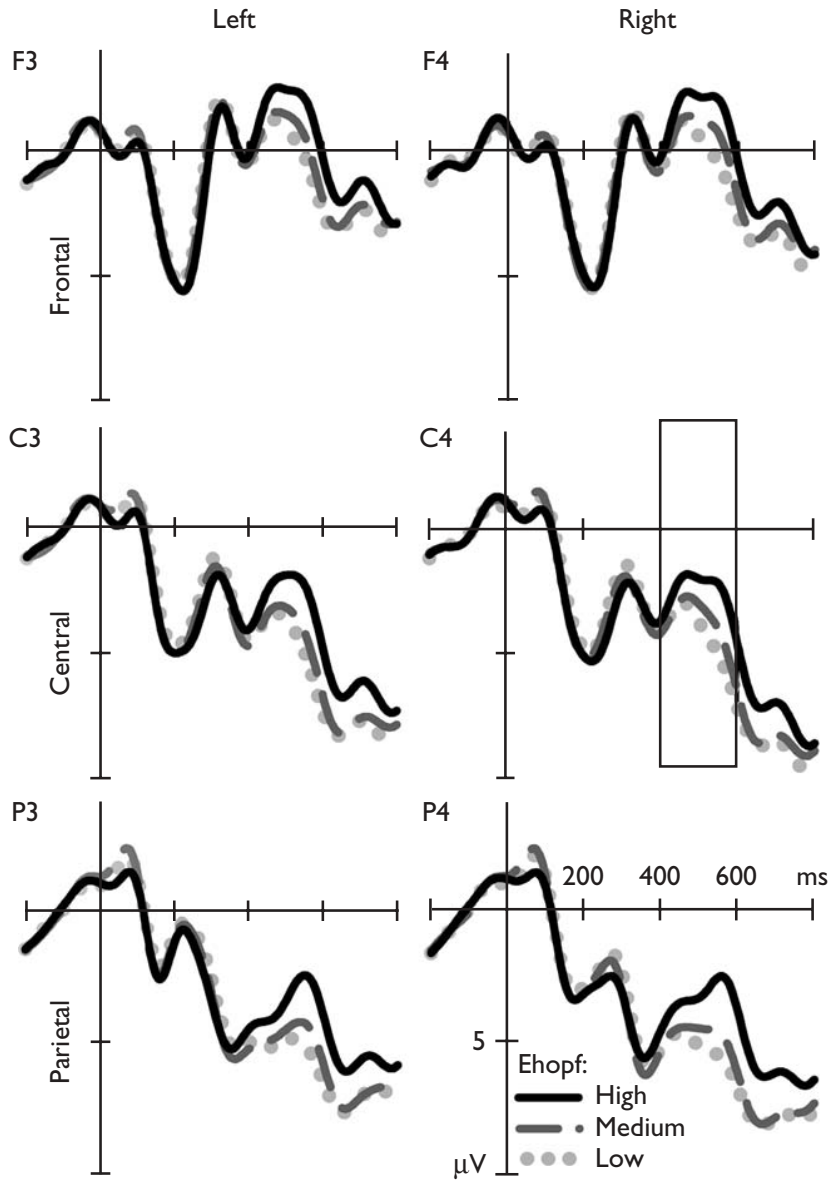

Fig. 2 N2 amplitudes increase with increasing levels of Hopfield Energy (Ehopf), as a measure of conflict. This conflict-monitoring effect resulting from nonword processing is indicated at six representative electrodes, and its time frame $(400-600 \mathrm{~ms})$ is indicated by the box at the $\mathrm{C} 4$ electrode.

and MUND). Three categories, were generated by categorizing the 100 stimuli with the lowest, medium, and the highest Ehopf values into three stimulus categories, respectively.

Stimuli were presented as black uppercase letters (courier font, about $4 \times 1 \mathrm{~cm}$ ) on a light grey screen of a 17-inch color monitor $(1024 \times 768$ pixels, $75 \mathrm{~Hz})$. Distance from eye to monitor was about $70 \mathrm{~cm}$. Stimuli were presented by ERTS software (BeriSoft Corp., Frankfurt, Germany) in a pseudorandomized fashion. No more than three words or nonwords were allowed to appear consecutively. After presenting 30 practice trials, stimuli were presented in six blocks of 100 trials. The participants were instructed to respond as fast as possible but not at the expense of accuracy. Half of the participants were instructed to press the right button to words and the left button to nonwords, the other half vice versa.

Each trial began with fixation points $\left({ }^{\prime}{ }^{\prime}\right)$ presented for $500 \mathrm{~ms}$, followed by the stimulus for $100 \mathrm{~ms}$. Thereafter, a mask ('\#\#\#\#') was presented for $300 \mathrm{~ms}$, followed by a blank screen that remained until a response was given (maximally $4 \mathrm{~s}$ ). After a 1.2-s pause, participants were instructed to press a button to start with the next trial, starting after a 1-s delay. 


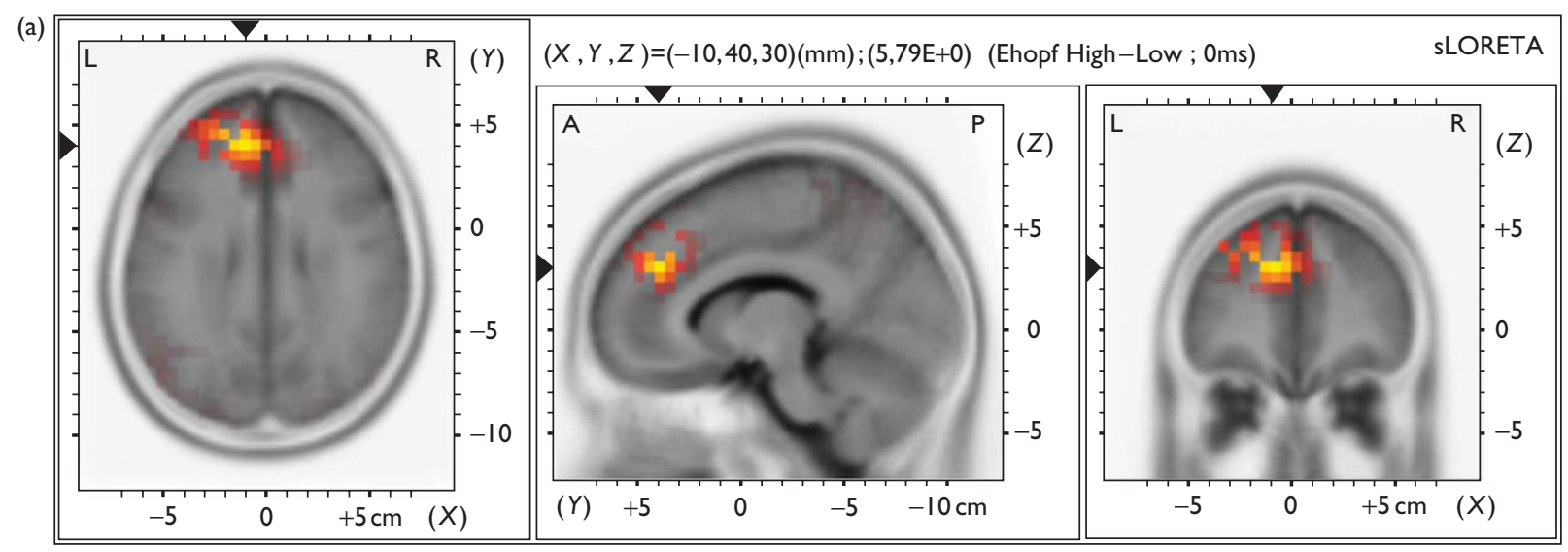

(b)

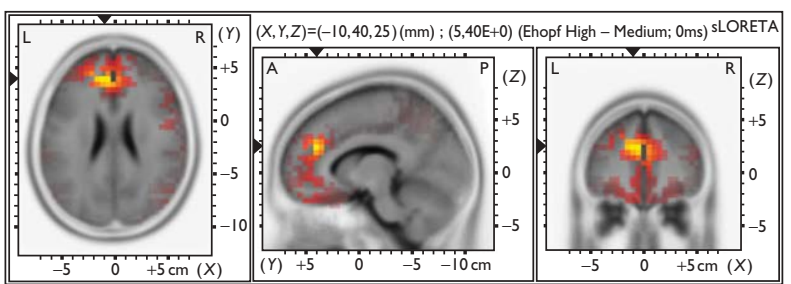

(c)

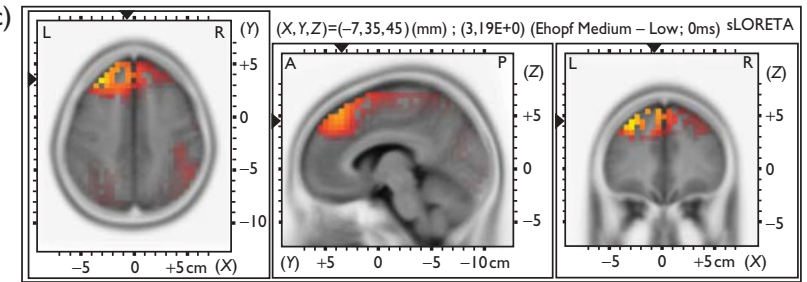

Fig. 3 Results of the standardized low-resolution brain electromagnetic tomography (sLORETA) analysis comparing the source density of the N2 according to different Hopfield Energy (Ehopf) levels: (a) high-low, (b) high-medium, and (c) medium-low. The X-Y-Z coordinates (MNI space) in each panel correspond to the respective maximum activations in the medial frontal gyrus.

Electroencephalogram data were recorded using ANT Software (ANT Software, B.V., Enschede, The Netherlands), and analyzed by Brain Vision Analyzer software (Brain Products $\mathrm{GmbH}$, Gilching, Germany). The participants were seated in an acoustically shielded chamber. Twenty-six electrodes were attached to an elastic cap (Easy Cap Corp., Herrsching-Breitbrunn, Germany) at 10-20 positions (T7, FT7, FT8, F7, C3, FC3, F3, FP1, FZ, FP2, FC4, F4, AFZ, F8, CP5, P3, P7, O1, PZ, CZ, C4, P4, O2, CP6, T8, and P8) and referenced to the left mastoid. Bipolar electrodes were attached above and below the right eye for the vertical electrooculogram and at the outer canthus of each eye for the horizontal electrooculogram. Impedances for the scalp and mastoid electrodes were kept below $5 \mathrm{k} \Omega$ for reference and active electrodes, and below $20 \mathrm{k} \Omega$ for eye-movement electrodes. Electroencephalogram data were sampled at $250 \mathrm{~Hz}$ and band-pass filtered $(0.1-30 \mathrm{~Hz})$.

Incorrect responses and outliers (response times faster than 300 or slower than $1600 \mathrm{~ms}$ ) were excluded from the analyses. Muscle artifacts, drifts, amplifier blockings, and eye movements were rejected by visual inspection. Blink artifacts were corrected using independent-component analysis [10].

For participant analyses, single participant averages were calculated for the three Ehopf categories separately using segments from $200 \mathrm{~ms}$ before the stimulus to $1 \mathrm{~s}$ after the stimulus, and baseline corrected ( $200 \mathrm{~ms}$ before stimulus). Grand averages were $8 \mathrm{~Hz}$ low-pass filtered for presentation purposes.

The ERP morphology (Fig. 2) showed a P1 peak at around 150-200 ms. A negativity followed the peaking at around 200-300 ms (N1). The P2 peaked around 300-400 ms and was immediately followed by a negative deflection peaking from 400 to $600 \mathrm{~ms}$ (N2). The N2 was most pronounced at the frontal sites, and showed a graded increase in negativity with increasing Ehopf in all electrodes. N2 amplitudes (400-600 ms) were averaged across electrodes for each condition, submitted to repeated-measures analyses of variance, and Greenhouse-Geisser corrected when the sphericity assumption was violated.

Single participant averages of the N2 were subjected to sLORETA [8], and normalized participant-wise. Paired $t$-tests were conducted on each possible pair of conditions (high vs. low Ehopf, high vs. medium Ehopf, and medium vs. low Ehopf). Statistical testing was performed on average source density. $t$-Tests were performed using sLORETA randomization procedure to correct for multiple comparisons. Variance smoothing was set to one.

Ehopf was used as the predictor for response times, sum of errors per item, and mean amplitudes over all channels. Analyses were conducted on all items for which at least 10 observations remained after outlier, error, and artifact rejections. This resulted in 204 items remaining for item analyses.

\section{Results}

The ERP participant analysis revealed a significant Ehopf effect $[F(1.4,17.9)=5.3, P \leq 0.05$, see Fig. 2]. High, medium, and low Ehopf nonwords revealed a mean amplitude of 3.1 (SD: 1.2), 3.5 (SD: 1.5), and $3.9 \mu \mathrm{V}$ (SD: 1.6), respectively.

sLORETA analysis revealed the largest $t$-value in the high Ehopf vs. low Ehopf contrast in a medial frontal gyrus voxel $(t=5.8, P \leq 0.005$; Montreal Neurological Institute (MNI) $X$ : $-10, Y: 40, Z: 30$, see Fig. 3). The significant region extended to the adjacent anterior cingulate cortex $(t=4.9, P \leq 0.05$; MNI X: -7, Y: 36, Z: 30). The largest Ehopf high vs. Ehopf medium contrasts were obtained in the medial frontal gyrus $(t=5.4, P \leq 0.005$; MNI $X:-10, Y: 40, Z: 25)$ and the anterior cingulate cortex $(t=4.8, P \leq 0.05$; MNI $X:-4, Y: 35, Z: 22)$. 
The largest difference between the Ehopf medium and the Ehopf low condition was obtained in the left middle frontal gyrus ( $t=4.17, P \leq 0.1$; MNI $X:-30, Y: 35, Z: 45)$, extending to other nonsignificant differences in the medial frontal gyrus ( $t=2.86$; MNI $X:-11, Y: 31, Z: 45)$. In the item analysis, Ehopf accounted for $12 \%$ of the $\mathrm{N} 2$ amplitude variance $(P \leq 0.001)$.

The participant analyses of behavioral data revealed a significant Ehopf effect in response times $[\mathrm{F}(2,26)=83.4$, $P \leq 0.001]$ and errors $[F(2,26)=22.1, P \leq 0.001]$. High, medium, and low Ehopf nonwords revealed a mean response time of 888 (SD: 143), 831 (SD: 136), and $784 \mathrm{~ms}$ (SD: 121), and error rates of 13.9 (SD: 9.7), 8.1 (SD: 7.4), and 5.9 (SD: 5.7), respectively. In the item analysis, Ehopf accounted for $28 \%$ of the response time and $5 \%$ of the error variance $(P \mathrm{~s} \leq 0.001)$.

\section{Discussion}

The participant analysis of ERP data revealed an ERP effect of Ehopf at all electrodes (see Fig. 2). This effect was apparent in the $\mathrm{N} 2$, and occurred at a relatively late time frame (400-600 ms) in comparison with other N2 effects. The error-related negativity, however was proposed to be another example for a rather late N2. This was demonstrated by using Ehopf to predict the amount of conflict, and supported by the anterior cingulate cortex being the common source of both effects [7]. The error-related negativity occurs after the response, which is usually later than $400 \mathrm{~ms}$ after the stimulus.

To examine whether the N2 in this study is functionally equivalent to the earlier N2s, we conducted sLORETA source localization. High Ehopf nonwords elicited a larger source density in the anterior cingulate cortex and the medial frontal gyrus than medium and low Ehopf nonwords. Botvinick et al.'s [1] hypothesis has, thus, been confirmed: greater anterior cingulate cortex activation was observed with increasing levels of conflict. The pivotal role of the medial frontal gyrus was demonstrated as well [2]. The extent of conflict between lexical representations modeled by Ehopf predicted these source-localization findings: thus this study suggests the functional equivalence of previous $\mathrm{N} 2$ findings and the current one.

This proposal is corroborated by the item analysis of the N2. Ehopf accounted for $12 \%$ of the ERP variance, which seems to be a good score for item-based ERP analyses $[11,12]$. The quantifiable model-to-data fit allows for competition between different models with respect to the variance explained [13], as previously proposed for behavioral data [14]. Ehopf accounted for a significant portion of response time $\left(R^{2}=0.28\right)$ and error variance $\left(R^{2}=0.05\right)$, suggesting that competing activated word representations delay the response times and result in higher error rates.

The current N2 time window is largely similar to the time window of N400 [15]. It was demonstrated that with an increasing number of orthographic neighbors, the N400 increases to nonwords $[16,17]$. Orthographic neighbors are words that are identical to the stimulus with respect to all but one letter (e.g. rose is an orthographic neighbor of nose). Holcomb et al. [17] interpreted increased response times, error rates, and N400s in terms of an increased lexical activation to nonwords with many orthographic neighbors. Grainger and Jacobs' lexical-inhibition hypothesis suggests that the competition between activated orthographic neigh- bors can inhibit behavioral responses [3]. To test whether Ehopf can contribute to the N400 discussion of orthographic neighborhood, we confirmed the assumption that the variance of the number of orthographic neighbors is contained in the Ehopf variance $\left(R^{2}=0.52, P \leq 0.001\right)$. Ehopf, however, accounted for an ERP variance of the N2 $\left(R^{2}=0.12\right)$ that was twice the number of orthographic neighbors $\left(R^{2}=0.06, P \leq 0.001\right)$; therefore, we propose that the competition between activated word representations contributes to the N400. As the MROM simulates word frequency effects by setting the resting levels of the word nodes accordingly, high-frequency word nodes are more strongly activated than low-frequency word nodes. Therefore, Ehopf is larger when high-frequency words compete. This is consistent with previous behavioral [3] and neurobiological [1,6] findings demonstrating the amount of competition to be dependent on the frequency of the word representations, and can account for the variance gain of Ehopf in comparison with the number of orthographic neighbors alone.

The current N2 finding is consistent with previous N2 findings in conflict monitoring, and with N400 findings in the psycholinguistic literature. This suggested functional overlap seems to confirm Polich's [18] notion that both are 'a reflection of the system's overall capability to comprehend complex similarities and relationships among stimulus items' (p. 319).

\section{Conclusion}

Response times, errors, the N2, and mediofrontal cortex activity were increased with the simulated extent of conflict between word nodes of the MROM. This is consistent with the conflict-monitoring theory, and might suggest a common functional locus of the N2 and the N400 in lexicalprocessing tasks.

\section{Acknowledgement}

This work was partly supported by a grant of the Deutsche Forschungsgemeinschaft (research unit 'conflicts as signals in cognitive systems', TP 3 Jacobs, JA 823/4-1).

\section{References}

1. Botvinick MM, Braver TS, Barch DM, Carter CS, Cohen JD. Conflict monitoring and cognitive control. Psychol Rev 2001; 108:624-652.

2. Ridderinkhof KR, van den Wildenberg WPM, Segalowitz SJ, Carter CS Neurocognitive mechanisms of cognitive control: the role of prefrontal cortex in action selection, response inhibition, performance monitoring, and reward-based learning. Brain $\operatorname{Cogn}$ 2004; 56:129-140.

3. Grainger J, Jacobs AM. Orthographic processing in visual word recognition: a multiple read-out model. Psychol Rev 1996; 103: 518-565.

4. Jacobs AM, Rey A, Ziegler JC, Grainger J. MROM-p: An interactive activation, multiple readout model of orthographic and phonological processes in visual word recognition. In: Grainger J, Jacobs AM, editors. Localist connectionist approaches to human cognition. Mahwah, New Jersey: Lawrence Erlbaum Associates Inc.; 1998. pp. 147-188.

5. Botvinick MM, Cohen JD, Carter CS. Conflict monitoring and anterior cingulate cortex: an update. Trends Cogn Sci 2004; 8:539-546.

6. Thompson-Schill SL, D'Esposito M, Aguirre G, Farah M. Role of left inferior prefrontal cortex in retrieval of semantic knowledge: a reevaluation. Proc Natl Acad Sci U S A 1997; 94:14792-14797.

7. Yeung N, Botvinick MM, Cohen JD. The neural basis of error detection: conflict monitoring and the error-related negativity. Psychol Rev 2004; 111:931-959. 
8. Pascual-Marqui RD. Standardized low-resolution brain electromagnetic tomography (sLORETA): technical details. Methods Find Exp Clin Pharmacol 2002; 24 (Suppl D):5-12.

9. Jacobs AM, Graf R, Kinder A. Receiver operating characteristics in the lexical decision task: evidence for a simple signal-detection process simulated by the multiple read-out model. J Exp Psychol Learn Mem Cogn 2003; 29:481-488.

10. Onton J, Westerfield M, Townsend J, Makeig S. Imaging human EEG dynamics using independent component analysis. Neurosci Biobehav Rev 2006; 30:808-822.

11. Dambacher M, Kliegl R, Hofmann M, Jacobs AM. Frequency and predictability effects on event-related potentials during reading. Brain Res 2006; 1084:89-103.

12. Hutzler F, Bergmann J, Conrad M, Kronbichler M, Stenneken P, Jacobs AM. Inhibitory effects of first syllable-frequency in lexical decision: an event-related potential study. Neurosci Lett 2004; 372:179-184.
13. Jacobs AM, Grainger J. Models of visual word recognition: sampling the state of the art. J Exp Psychol Hum Percept Perform 1994; 20: 1311-1334.

14. Perry C, Ziegler JC, Zorzi M. Nested incremental modeling in the development of computational theories: the CDP + model of reading aloud. Psychol Rev 2007; 114:273-315.

15. Kutas M, Federmeier KD. Electrophysiology reveals semantic memory use in language comprehension. Trends Cogn Sci 2000; 4:463-470.

16. Braun M, Jacobs AM, Hahne A, Ricker B, Hofmann M, Hutzler F. Modelgenerated lexical activity predicts graded ERP amplitudes in lexical decision. Brain Res 2006; 1073-1074:431-439.

17. Holcomb PJ, Grainger J, O'Rourke T. An electrophysiological study of the effects of orthographic neighborhood size on printed word perception. J Cogn Neurosci 2002; 14:938-950.

18. Polich J. Semantic categorization and event-related potentials. Brain Lang 1985; 26:304-321. 